\title{
Video holográfico en el dominio de Fresnel, objetos 3D
}

\author{
Dudbil O Pabon-R, Jorge-Enrique Rueda-P
}

Grupo Óptica Moderna, Departamento de Física, Universidad de Pamplona, Pamplona, Colombia

\section{Resumen}

Generamos Video-Holográfico en el dominio de Fresnel y para objetos de volumen en movimiento. Implementamos un arreglo óptico tipo Mach-Zenhder y desarrollamos la herramienta computacional HDFresnel_V1.exe. Esta herramienta permite registrar y reconstruir hologramas, mejorar la calidad del holograma reconstruido, y generar videohologramas.

Palabras clave: Holografía, Transformada de Fresnel digital, Video.

\section{Video holographic Fresnel domain, 3D-objects}

\begin{abstract}
Holographic video at the domain Fresnel of 3D moving objects was obtained. For this purpose an optical Mach-Zenhder type arrangement was implemented and developed HDFresnel_V1.exe computational tool. This tool allows you to record holograms, holograms reconstruct, improve the quality of the reconstructed hologram, and generate hologramsvideo.
\end{abstract}

Keywords: Holography, Digital Fresnel Transform, Video.

*Para citar este artículo: Pabon-R D, Rueda-P J E. Video holográfico en el dominio de Fresnel, objetos 3D. Revista Bistua. 2016.14(1):32-37

+ Autor para el envió de correspondencia y la solicitud de las separatas: Rueda-P,Jorge-E., Grupo Óptica Moderna, Departamento de Física, Universidad de Pamplona, e-mail: jorgeenriquerueda@gmail.com 
33

\section{Introducción}

La holografía, propuesta por Dennis Gabor $^{1}$, es una técnica de modulación de una onda objeto en la fase y amplitud de un patrón de franjas de interferencia, el cual se genera mediante la superposición entre la onda objeto y una onda de referencia.

Goodman $^{5}$ y Lawrence ${ }^{2}$ propusieron la holografía digital, esta técnica consiste en hacer un registro digital del holograma óptico, y hacer la reconstrucción digital u óptica. Los avances logrados hasta hoy, apilan un buen número de aplicaciones, entre ellas, microscopía holográfica, análisis de partículas, sistemas microelectromecánicos, metrología holográfica, entre otras. Una de las tendencias actuales de la holografía es la construcción de videohologramas.

En esta investigación se implementó la técnica de holografía digital de Fresnel para objetos de volumen, usando un arreglo Mach-Zehnder (Figura 1), el cual permite generar fotogramas y construcción del videoholográfico.

\section{Método y materiales}

La imagen en la Figura 1 corresponde al arreglo óptico implementado.

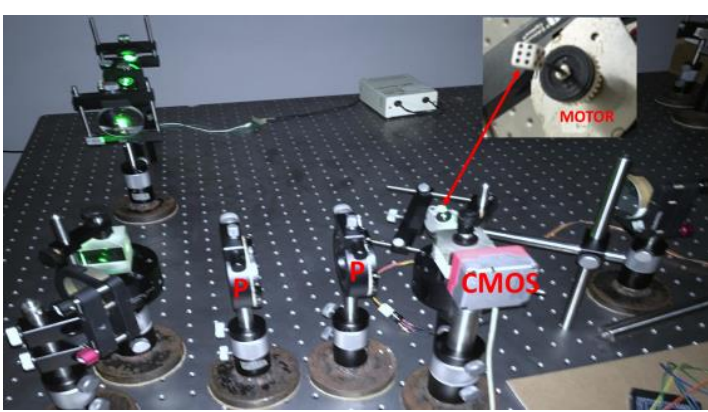

Figura 1. Imagen del arreglo holográfico Mach-Zehnder. La fuente de iluminación es un puntero Laser de $532 \mathrm{~nm}$ de longitud de onda. CMOS es una cámara digital de 1944x2592 pixeles, con tamaño de pixel de 2,2 $\mu \mathrm{m}$. P: polarizadores lineales. Objeto: un dado de dimensiones $5 \times 5 \times 5 \mathrm{~mm}^{3} . \quad z=12 \mathrm{~cm}$ es la distancia entre el objeto y el plano del holograma (plano de la CMOS).

Mediante la cámara CMOS se obtiene el holograma digital del objeto. Se trata de un holograma de Fresnel por cuanto grabamos el patrón de interferencia producido por la superposición entre el haz de referencia y el haz objeto, siendo este último el patrón de difracción de Fresnel del objeto (un dado) a $z=12 \mathrm{~cm}$. Para efecto de la reconstrucción del holograma, se desarrolló una herramienta computacional que reconstruye el holograma mediante la transformada de Fresnel digital. Esta misma herramienta puede operar en modo CPU 0 GPU, permite registrar fotogramas, reconstruirlos $y$ empaquetarlos en formato de video. 
34

\section{Transformada Digital de Fresnel (TDFr)}

En este trabajo implementamos un algoritmo de la TDFr para la reconstrucción del holograma. Esta transformada tiene la forma $3,4,6,7,8,9,10,11$ :

$$
\begin{aligned}
& \mathrm{U}\left(\mathrm{k} \Delta \mathrm{x}^{\prime}, \ell \Delta \mathrm{x}^{\prime}, \mathrm{z}\right)=\frac{1}{\mathrm{i} \lambda \mathrm{z}} \mathrm{e}^{\mathrm{i} \frac{2 \pi}{\lambda z}\left(\left(\mathrm{k} \Delta \mathrm{x}^{\prime}\right)^{2}+\left(\ell \Delta \mathrm{y}^{\prime}\right)^{2}\right)} . \\
& \cdot \operatorname{IDFT}\left\{\mathrm{h}(\mathrm{m} \delta \mathrm{x}, \mathrm{n} \delta \mathrm{y}, 0) \mathrm{e}^{\mathrm{i} \frac{2 \pi}{\lambda z}\left((\mathrm{~m} \delta \mathrm{x})^{2}+(\mathrm{n} \delta \mathrm{y})^{2}\right)}\right\}
\end{aligned}
$$

donde IDFT representa la transformada digital de Fourier inversa, $\lambda$ es la longitud de onda de la luz utilizada, $k, \ell, m$, y $n$ son números enteros. $\quad h(m \delta x, n \delta y, 0)$ es la transmitancia del holograma que se reconstruye mediante la Ec.(1), para una distancia $z$; siendo esta distancia entre el objeto y el medio de registro del holograma (CMOS). La reconstrucción del holograma es una matriz de $N \times M$ puntos con un periodo de muestreo $\Delta y^{\prime}$ y $\Delta x^{\prime}$, respectivamente.

La Figura 2 muestra una imagen de la interfaz de usuario para el manejo de la herramienta computacional implementada. La interfaz está constituida de un bloque de cuatro cuadrantes imagen (1: imagen de salida de la cámara de video ubicada en el plano del holograma óptico; 2: imagen del holograma registrado por la cámara de video; 3: imagen de la reconstrucción del holograma; 4: imagen de reconstrucción del holograma sometido a filtrado); el bloque 6 es de parámetros de control de la cámara de registro; el bloque 7 para ingreso de las características de la cámara de registro, longitud de onda de la luz utilizada y la distancia entre el objeto y el plano de la cámara de registro. El bloque 8 es una herramienta de manipulación del registro y reconstrucción del holograma, de tratamiento del holograma. El bloque 9 permite guardar las imágenes de los cuadrantes 2 al 4 , y cargar hologramas almacenados previamente.

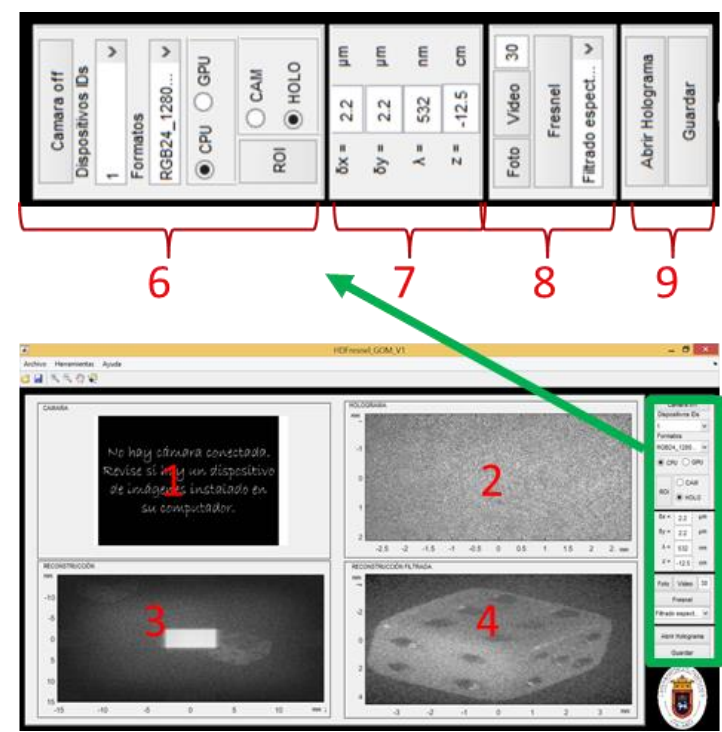

Figura 2. Interfaz de usuario de la herramienta HDFresnel_GOM_V1.exe. La herramienta permite trabajar sobre CPU o GPU; mediante esta última opción el procesamiento se realiza 


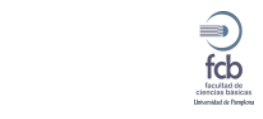

35

en paralelo mejorando notablemente el costo computacional; para las características del computador utilizado, se determinó que procesar un holograma de 2592x2592 pixeles, en la CPU toma un tiempo de 656.1 $\mathrm{ms}$, mientras que con la GPU es de $38.7 \mathrm{~ms}$, es decir aproximadamente el $6 \%$ del tiempo utilizado por la CPU.

\section{Resultados y discusión}

Las imágenes en la figura 3(a) son un resultado de la distribución de intensidad de un holograma del dado; el tamaño de la imagen es de $1944 \times 2592$ pixeles. La figura 3(b) es la reconstrucción digital del holograma (Fig.3(a)), esta reconstrucción es la imagen de la transformada de Fresnel del holograma a la distancia $Z=12 \mathrm{~cm}$. La figura 3(c) es la reconstrucción del holograma 3(a) después de aplicar un filtro mediana. La Fig.3(d) es la reconstrucción del holograma después de aplicar un filtro pasa-alto.

La Fig. 4 muestra tres fotogramas del video del dado en movimiento angular de $60 \mathrm{rpm}$. La duración del video es de 30 s, y se grabó a una frecuencia de 16 cuadros/segundo. El video se obtuvo trabajando en modo GPU. Este resultado demuestra que es posible la generación de videoholográfico de fenómenos dinámicos.

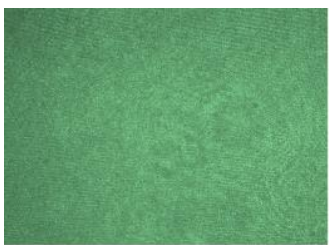

(a)

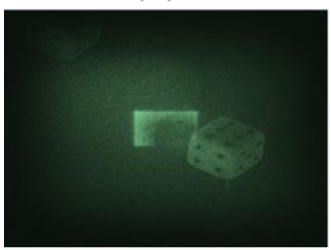

(c)

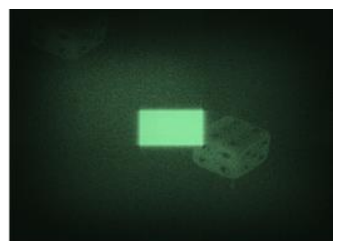

(b)

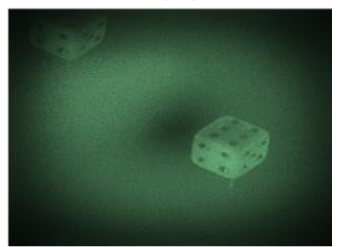

(d)
Figura 3. Resultados experimentales. (a) Holograma digital del dado; (b) Reconstrucción digital del holograma (a); (c) Reconstrucción digital de (a) después de aplicar un filtro mediana; (d) Reconstrucción de (a) después de aplicar un filtro pasa alto.

Las perspectivas están pensadas en superar el tiempo de registro de cada fotograma, y así obtener videos de mayor frecuencia que 16 cuadros/segundo.

La frecuencia de grabación se podría aumentar reduciendo el tamaño de cada fotograma, pero esto implica un costo en pérdida de resolución. El peso del video es otro problema por resolver; el video obtenido de 30 segundos y tiene un peso de 35.5 MB. 


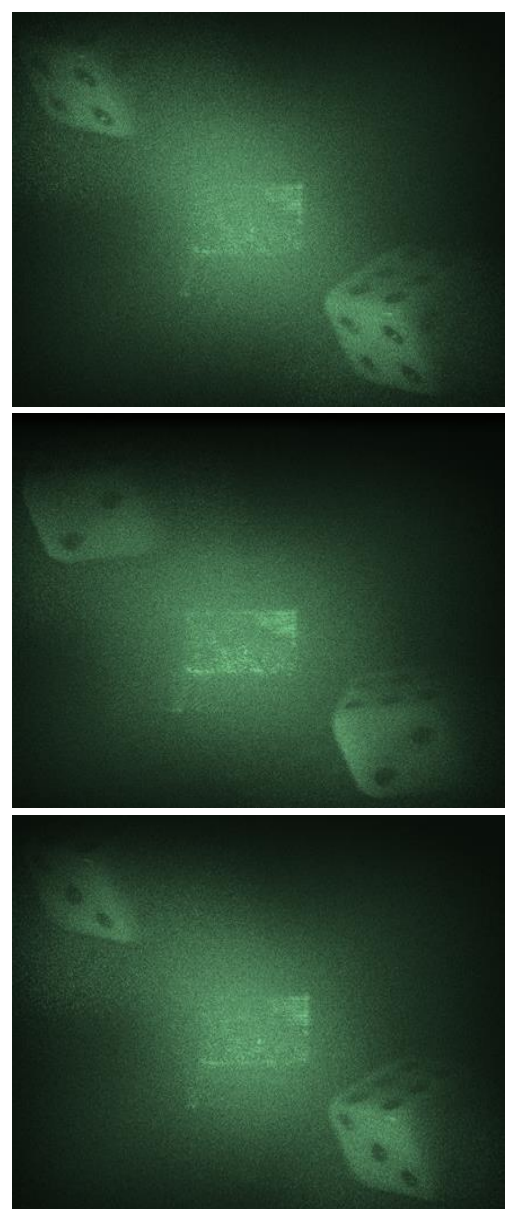

Figura 4. Secuencia de fotogramas del video holográfico empaquetado a 16 Cuadros/Segundo, del movimiento del dado girando a 60rpm.

\section{Referencias bibliográficas}

1. Gabor D. A new microscopy principle. Nature Publishing Group. 1948 (161): 777-778.

2. JWG R, Lawrence W. Digital image formation from electronically detected holograms. Applied physics Letters. 1967 (11)77: 77-79.

3. Li J, Picart P. Digital Holography. United States: John Wiley \& Sons. 2012.
4. Mihaylova E. Holography-Basic Principles and Contemporary Applications. Intech. 2013.

5. Goodman JW. Introduction to Fourier Optics. Greenwood Village: Roberts and Company Publishers. 2005 (3).

6. Schnars U, Jueptner W. Digital Hologram Recording, Numerical Reconstruction, and Related Techniques. New York: Springer Berlin Heidelberg. 2005.

7. Poon TC, Liu JP. Introduction to modern digital holography with MATLAB. New York: Cambridge University Press. 2014.

8. Kreis TM, Adams M, Jueptner WPO. Methods of digital holography: a comparison. Spie Proceedings. 1997 (3098): 224-233.

9. Schnars U, Falldorf C, Watson J. Digital Holography and Wavefront Sensing; Principles, Techniques and Applications, Second ed., Berlin Heidelberg: Springer-Verlag. 2015.

10. Ma Z, Yang $Y$, Ge Qi, Deng L, Xu Z, Sun $X$. Nonlinear filtering method of zero order term suppression for improving the image qualityin off-axis holography. Optics Communications. 2014 (315): 232-237.

11. Qiu H, Gu J. Elimination of zero-order and conjugate images in offaxisdigital holography. Optik. 2014:2652-2655, 2014. 
37

Pabon-R D.O.

Físico-MSc..en Física. Universidad de Buenos Aires, Argentina.
Rueda-P,Jorge-E:

Ph.D.Profesor Titular. Investigador Asociado. Grupo Optica Moderna. Departamento de Fisica.Universidad de Pamplona-Colombia 